\title{
Review of the early discharge summary in the Grampian area
}

\author{
Shona A. Walker and John M. Eagles
}

\begin{abstract}
The psychiatric discharge summary is an important means of communication between general practitioners (GPs) and psychiatrists and invaluable in the continulty of patient care. The earty discharge summary therefore needs to provide relevant information for GPs during this vulnerable period. We surveyed GPs in the Grampian area in order to obtain information felt to be most relevant and the results were incorporated into a now semi-structured format which is now currently being used in the Grampian area.
\end{abstract}

The letter is the main instrument of communication between specialists and GPs. The psychiatric discharge summary is one important aspect of this communication. Craddock (1990) looked at the information contained in the formal discharge letter and concluded that prognosis, advice about management and information given to the patient are often poorly covered, although these items are rated as important by GPs. Craddock \& Craddock (1989) also looked at the use of different formats for such a summary and found a discrepancy between the preference of GPs and psychiatrists. Psychiatrists tend to prefer a lengthy summary conforming to the Institute of Psychiatry guidelines, whereas GPs prefer a shorter summary containing pertinent information. Rigby (1988) found that two thirds of GPs were in favour of a semi-structured format with priority being given to diagnosis and prognosis. It was also felt that this would ensure the inclusion of important items and limit the time and effort spent on less important ones. Orrel \& Greenberg (1986) found that $98 \%$ of GPs wanted a brief communication of events within two weeks of patients' discharge, but only $26 \%$ of GPs received such a service.

The psychiatric discharge summary has also been suggested as a useful tool for management and audit (Essex \& Doig, 1991). By identifying the local information needs of GPs and incorporating this into a relevant format it was felt that the continuity of care could be improved. This format could also be used to audit various aspects of psychiatric practice and provide information for GPs, psychiatrists and other members of the psychiatric and primary care teams relevant to shared patient care.

\section{The study}

The aim of the study was to review the information needs of GPs in the Grampian area in relation to the discharge of psychiatric patients and to design an early discharge summary to meet these requirements. The Grampian area has just over 300 GPs looking after a mixed urban and rural population. The current early discharge summary in the Grampian area is used for all hospital specialties and it was felt that its format could be modified to be more specific to the psychiatric services.

A questionnaire was devised incorporating 24 different items that could be included in the summary, as follows.

(1) Discharge mental state examination.

(2) Physical examination.

(3) Diagnosis.

(4) Legal status.

(5) Investigations.

(6) Premorbid personality.

(7) Prognosis.

(8) Data of admission.

(9) Discharge treatment.

(10) Information given to the patient.

(11) Personal history.

(12) Date of discharge.

(13) Past medical history.

(14) In-patient treatment.

(15) Admission mental state examination.

(16) History of presenting complaint.

(17) Family history.

(18) Medication on admission.

(19) In-patient progress.

(20) Alcohol.

(21) Past psychiatric history.

(22) Reason for admission.

(23) Advice regarding management.

(24) Drug abuse.

GPs were then asked to prioritise their information needs. Also included in the questionnaire were questions about the current discharge policy which involves the early discharge summary being handed to the GP by the patient. A range of other options were listed as alternatives 
Table 1. Top ten items identified by GPs for inclusion in an early discharge summary

\begin{tabular}{ll}
\hline $85 \%$ & Diagnosis \\
$84 \%$ & Discharge treatment \\
$72 \%$ & Mental state examination at discharge \\
$71 \%$ & Date of discharge \\
$70 \%$ & Advice regarding management \\
$67 \%$ & In-patient treatment \\
$65 \%$ & Prognosis \\
$62 \%$ & Information given to the patient \\
$52 \%$ & Date of admission \\
$47 \%$ & Reason for admission \\
\hline
\end{tabular}

combining the current system with telephone contact and direct posting to the GP. They were also asked their opinion about ward receptionists making GP appointments for patients at the time of their discharge.

The questionnaire was sent to a random sample of 55 GPs in the Grampian area. A postal reminder was sent after one month with a telephone reminder two weeks later.

\section{Findings}

The response rate to the questionnaire was 40 out of 55 GPs (72\%). From their responses, the top ten items for inclusion in an early discharge summary (in order of summated ranking and with the percentage of GPs selecting each of these items) is illustrated in Table 1.

With regard to the current policy of asking patients to hand-deliver their letter to the GP, $70 \%$ of GPs felt this to be a good system. Of the other options described, 38\% preferred both hand-delivery and telephone communication. $17 \%$ preferred postal delivery with $12 \%$ also re questing hand-delivery of a copy, $15 \%$ preferred only hand-delivery, and $7 \%$ requested only telephone contact. Postal delivery along with telephone communication was the option of $5 \%$ of GPs and $2 \%$ recommended a combination of all three variables. Some form of telephone contact was requested by $52 \%$ of GPs, $71 \%$ of GPs felt it would be very useful for the ward receptionist to phone for a GP appointment at the time of discharge whereas $29 \%$ felt this was not appropriate.

\section{Comments}

The first two weeks after discharge is a vulnerable period for many psychiatric patients. Therefore the early discharge summary needs to provide relevant information in order to ensure effective continuity of care. Our response rate of $72 \%$ suggested that we had obtained the opinions of a representative sample of GPs. Our findings regarding GPs' preferences are comparable with other studies which have looked at the use of the formal discharge summary. The early discharge summary plays an important role in ensuring continuity of care, especially in view of the time that the formal discharge summary may take to reach the GP. Although GPs seemed satisfied with the current format, there was felt to be room for significant modification. GPs requested a clear summary of diagnosis, discharge treatment, mental state examination at discharge, discharge date and advice regarding future management. This information is often not supplied routinely in the early discharge summary. It is noteworthy that $38 \%$ of GPs preferred hand-delivery of the early discharge summary plus telephone communication, this being the most popular option. The fact that $71 \%$ of GPs felt it would be useful if ward staff made GP appointments prior to discharge suggests that we should make this a standard practice under the current system.

Remodelling the format of the early discharge letter has been carried out, and this will comprise a single sheet of A4 on which the GPs' 'top ten items' will be included. An audit will later determine whether this revised format does indeed result in GPs' receiving more of the information they said they would welcome. The findings also suggest that telephone contact should be made with primary care at the time of discharge informing them of the discharge and arranging a GP appointment.

\section{References}

Craddock. N. (1990) Audit of psychlatric discharge summaries. Psychiatric Bulletin. 14, 618-620.

- \& CRADDOCK. B. (1989) Psychiatric discharge summaries - differing requirements of psychiatrists and GPs. British Medical Journal. 299, 1382-1384.

Essex. B. \& DoIG, R. (1991) The psychlatric discharge summary - a tool for management and audit. British Journal of General Practice, 41, 332-334.

ORREL. M. \& GREENBERG. M. (1986) What make psychiatric discharge summaries useful to GPs. Psychiatric Bulletin. 10. 107-109.

RigBY, J. (1988) Psychiatric discharge summaries - an assessment of need. The Practitioner, 282, 677-679.

Shona A. Walker, Registrar in Psychiatry; and John M. Eagles, Consultant Psychiatrist, Royal Cornhill Hospital, Cornhill Road, Aberdeen, AB9 2ZH 